\title{
Taxonomy of Source Code Security Defects Based on Three-Dimension-Tree*
}

\author{
Zhang Yan $^{1,2, * *}$, Dong Guowei ${ }^{2}$, Guo Tao $^{2}$, and Yang Jianyu ${ }^{3}$ \\ ${ }^{1}$ School of Computer Science and Engineering, Beihang University, Beijing, China \\ ${ }^{2}$ China Information Technology Security Evaluation Center, Beijing, China \\ ${ }^{3}$ China Agricultural University, College of Information and Electrical Engineering, \\ Beijing, China \\ zhangy@cse.buaa.edu.cn, \\ \{donggw, guotao\}@itsec.gov.cn, ycjyyang@126.com
}

\begin{abstract}
The authors present a new taxonomy for source code security defects based on three-dimension-tree, which considers the information of defect's cause, impact and representation synthetically. Case studies show that a sound system for classifying source code defects could be established with this taxonomy, and it is also good for the prevention and fixing of software vulnerabilities.
\end{abstract}

Keywords: three-dimension-tree, source code, security defect, taxonomy.

\section{Introduction}

Most security attacks are caused by the vulnerabilities of application system, and the defects that generated during software design and coding are the main source of them. Source code static analysis is an effective method for vulnerability reduction, because this method could consider the information of path widely and detect program's security defects automatically [1]. Generally, source code defects analysis includes lexical and syntax analysis, intermediate code generation and defect detection, as shown in Fig. 1. First, source code is compiled to Abstract Syntax Tree (AST) by lexical and syntax analyzer. Second, AST is transformed into Intermediate Representation (IR), such as Control Flow Graph (CFG), Call Graph (CF), and so on. Finally, IR is checked with defect detection rules and all kinds of analysis techniques, and the results are reported.

The construction of defect detection rule is an important step in above process, because the rule's description for detect affects analysis result directly. Classification of source code defects is helpful for the refinement of detection rules and the accuracy of analysis. Also, it is better for learning defects' nature and cause. Generally speaking, classification of source code defects is good for the prevention and fixing of new kinds of defects.

\footnotetext{
* This work was supported in part by the National Natural Science Foundation of China (90818021, 41171309).

** Corresponding author.
}

D. Li and Y. Chen (Eds.): CCTA 2013, Part I, IFIP AICT 419, pp. 232-241, 2014.

(C) IFIP International Federation for Information Processing 2014 


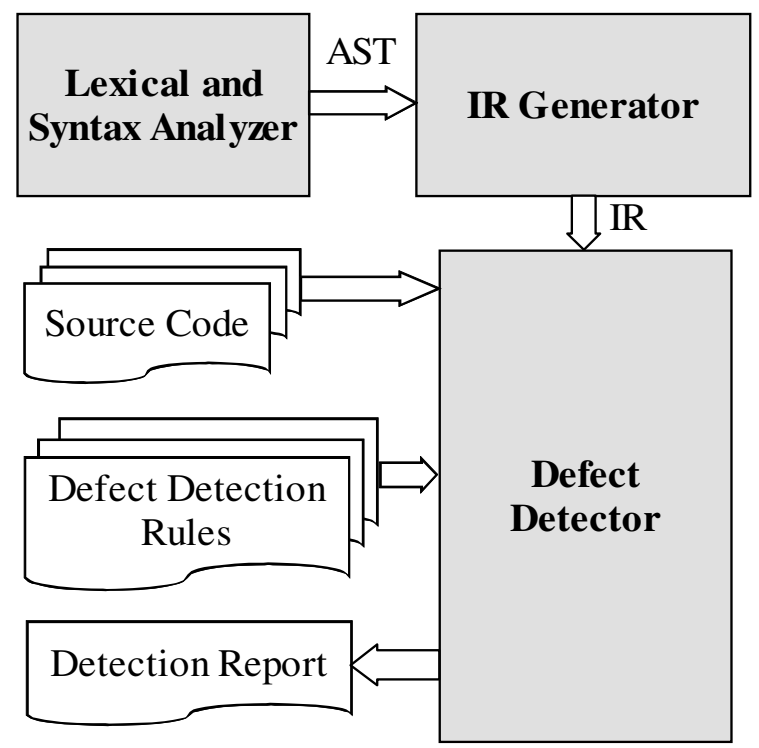

Fig. 1. Flow Graph of Source Code Defect Detection

At present, there is little taxonomy for source code defects, but the ones for software vulnerabilities are discussed by many researchers. In these works, vulnerabilities are sorted by cause [2-7], threat level [8], impact [4, 9-11], attack mode [5, 6, 11-15], fixing mode $[10,16]$, location $[4,5,17]$, and so on. Although they involve most aspects of vulnerabilities, they are not suitable for source code defects. The main reason is that most of these methods only focus on one attribute of vulnerability.

In this article, we first introduce existing taxonomies of software vulnerabilities, and then present a kind of taxonomy for source code defects based on three-dimension-tree, which considers the information of defect's generation cause, impact and representation synthetically. At last, we sort the defects that are listed in CWE [18] and Fortify [19] with this taxonomy. Case studies show that a sound system for sorting source code defects could be established with this taxonomy, and it is also a guide for the prevention and fixing of software vulnerabilities.

This paper is organized as follow: in section 2, existing taxonomies of software vulnerabilities are introduced; in section 3, the taxonomy of source code defects based on three-dimension-tree is presented; in section 4 , two case studies are given; in section 5, this paper is concluded.

\section{Overview of Software Vulnerability Taxonomy}

There are different definitions for software vulnerability as to different aspect, such as access control, state space, security strategy, etc [20]. Because of different requirement, existing taxonomies mostly focus on cause, impact, threat level, exploit mode, platform, and so on. 
Introduction of Existing Taxonomies. As for Unix system, T. Aslam provided a taxonomy of functional errors based on cause [3]. He divided Unix errors into 4 kinds: design error, environment error, coding error, and configuration error. Design errors are issues that are generated during requirement analysis and software design; Environment errors are caused by the limitation of operation environment, such as errors that are result from compiler or OS defects; Coding errors mainly include synchronization errors, condition verification errors, etc; Configuration errors mainly include install location errors, install parameter errors and install permission errors.

F.B. Cohen presented an attack-mode-oriented taxonomy [12]. He analyzed more than 100 attack sets, and sort vulnerabilities into 18 categories: error and missing, unused value, implicit trustable attack, data spoofing, process bypass, distributed coordination attack, input overflow, Trojan horse, error after data integration, incomplete daemon, unreleased function use, misuse by attack, prohibit audit, failure lead by increasing system load, using network services and protocols, inter-process communication attack, race condition, improper default value.

I. Krsul provided impact-oriented taxonomy [9]. He pointed out that the impact of vulnerability could be divided into direct impact and indirect impact, and he sorted software vulnerabilities into data access, command execution, code execution and denial of service.

As to multi-factor taxonomy, C.E. Landwehr presented a vulnerability taxonomy based on source, time and location, in which source indicates Trojan horse, back door, logic bomb, etc; time refers to the parse that vulnerability takes place in software development life cycle, such as design, coding, maintenance; location means OS level, support software level or hardware level [5]. Upon this work, K. Jiwnani provided a taxonomy based on cause, location and impact for abstracting issues in software development [4]. In his method, cause indicates validation error, domain error, sequence or alias error, etc; Location refers to system initialization, memory management, process management or scheduling; Impact means unauthorized access, root or system access, denial of service, and so on.

D. Wenliang presented a vulnerability-life-cycle-based taxonomy [10]. He defined vulnerability life cycle as the process of "Import-Damage-Fixing", and sorted vulnerabilities by cause, direct impact and fixing mode. Specifically, cause indicates input validation error, permission certificate error, sequence or alias error, etc; Direct impact refers to illegal code execution, illegal target change, illegal target resource access, denial of service, and so on; Fixing mode means entity false, entity missing, entity misplacing, entity error, etc.

CWE (Common Weakness Enumeration) is a defect dictionary provided by Mitre [18], which is used to provide a general criterion for identifying, reducing and preventing software defects. The last CWE version is 1.11 , and it includes more than 800 kinds of defects. In CWE, defects are sorted into 3 classes, which are code defects environment defects and configuration defects, and the code defects includes executable code defects, source code defects and the defects that violates security design principle. Further, source code defects are divided into 14 subclasses: data handling, API abuse, security features, time and state, error handling, indicator of poor code quality, channel and path errors, handler errors, web problems, user interface 
errors, initialization and cleanup errors, pointer issues, insufficient encapsulation. It's easy to see that the taxonomy for CWE's source code defects is based on cause.

Disadvantages of Existing Taxonomies. Based on the discussion above, we can see that there is little taxonomy for source code defects, and the ones for software vulnerability are not suitable for source code defects. The reasons are as follow:

(1) There is little taxonomy special for source code defects. Source code defects (or coding errors) are often defined as an independent category of software vulnerability, but are not divided deeply [3-5, 10]. In CWE, source code defects are only sorted into 14 simple classes [18];

(2) Some taxonomies could not be used for source code defects classification. For example, the ones based on attack mode or impact mostly consider the factors such as exploiting results $[9,12]$, which could not directly reflect the information of source code defects;

(3) Existing works could not reflect various aspects of defects. Most of them only consider one attribute of defect, such as cause, impact, which could only represent one aspect of defect. In addition, there are many overlaps between the categories that are generated by these methods. All of these are disbenefit for source code defect analysis.

\section{Taxonomy of Source Code Defects with Three-Dimension-Tree}

Classification Attributions of Source Code Defect. In order to regularize the process of source code defect analysis and provide wonderful defect detection rules, we research the taxonomy of source code defects. Based on the analysis above, vulnerabilities are sorted upon different attributes. Similarly, when classifying defects, we could also consider their attributes. After widely studying, we find that programmers often describe defects with their 6 attributes:

(1) Internal cause. This means the issues in source code that are generated during coding, such as the use of dangerous functions;

(2) Intended or unintended subjective cause. Intended defects are imported by developer deliberately, such as logic bomb and undeclared channel, and unintended defects are imported because of programmer's lack of the knowledge of secure coding;

(3) External cause. This attribute mainly focus on the issues generated by the call of external library, for which special environment should be considered;

(4) Impact of defects. This means the direct impact that is caused by source code defect, for example, buffer overflow;

(5) Issues arose in testing or execution. These are the error features that are shown in testing or running, such as I/O errors, calculation errors, logic errors, data handling errors, configuration errors, OS errors, interface errors, global variable errors, system crash, etc;

(6) Developing language. Some defects arise in special language. For example, J2EE configuration errors are special for Java.

We consider the complexity of defects, the extension of taxonomy and the 6 attributes above synthetically, and then provide 3 classification attributes for source code defects, which are cause, impact and representation: 
- $\quad$ Cause. This attribute includes the internal, external and subjective causes of defect generation. We have concluded 9 classes of defects in this aspect, which are input issues and validation, API errors, access control and password fail, share and race, exception handle, unsafe code, boundary treatment, configuration errors, malicious code. Details are shown in Tab. 1.

- Impact. This attribute is the direct impact that defect produces. We have collected 9 classes and more than 30 subclasses of defects in this aspect, such as overflow, injection, manipulation, web attack, access control, leak, file system, deadlock, and denial of service. Details are shown in Tab. 2.

- Representation. This means the form that defect presents in source code, which could also be seen as the form of code with issue, and secure issues may arise when running this code. Of course, some representations are related with special language. We have summarized 13 classes and more than 150 subclasses of defects in this aspect, such as pretreatment, declaration and initialization, expression, integer, float, array, string, memory management, input and output, object oriented, concurrency, as shown in Tab. 3.

Taxonomy Based on Three- Dimension-Tree.We consider 3 attributes when sorting source code defects. That is, the category of a defect is decided by its cause, impact and representation. From Tab. 1, 2, 3, we can see that all of the 3 classification attributes satisfy multi-level containment, which could be described with tree structure. So we represent each attribute with a tree, and the set that includes all leaf nodes of one tree is also the set of corresponding attribute's final categories. Details are shown in Fig. 2.

Definition (Three-Dimension-Tree Taxonomy). Let Tree $r e$, Tree $_{r t}$, and Tree Trp $_{r}$ be the trees that represent defect's cause, impact and representation, and child(n) the child node of node $n$ in tree, if $\operatorname{Re}=\left\{\right.$ rel $\left(\right.$ re $\left._{\text {Tree }}\right) \wedge \quad(\operatorname{child}($ re $\left.)==\mathrm{Null})\right\}$, $R t=\left\{r t \mid\left(r t \in\right.\right.$ Tree $\left.\left._{r t}\right) \wedge(\operatorname{child}(r t)==\mathrm{Null})\right\}$, and $R p=\left\{r p \mid\left(r p \in\right.\right.$ Tree $\left._{r p}\right) \wedge($ child $\left.(r p)==\mathrm{Null})\right\}$, then $\{(a, b, c) \mid(a \in R e) \wedge(b \in R t) \wedge(c \in R p)\}$ is the set of defect categories that are generated by the Three-Dimension-Tree Taxonomy, and each $(a, b, c)$ represents a category of defects.

Table 1. Causes of Defects

\begin{tabular}{|c|c|c|c|}
\hline \multirow{9}{*}{ Cause } & Intended & Malicious code & \\
\hline & \multirow{8}{*}{ Unintended } & \multirow{7}{*}{ Environment independent } & Input issues and validation \\
\hline & & & API errors \\
\hline & & & Access control and password fail \\
\hline & & & Share and race \\
\hline & & & Exception handle \\
\hline & & & Unsafe code \\
\hline & & & Boundary treatment \\
\hline & & Environment dependent & Configuration errors \\
\hline
\end{tabular}


Table 2. Impacts of Defects

\begin{tabular}{|c|c|c|}
\hline \multirow{22}{*}{ 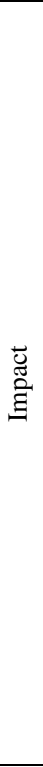 } & \multirow{4}{*}{ Injection } & SQL injection \\
\hline & & Command injection \\
\hline & & XML injection \\
\hline & & ..... \\
\hline & \multirow{3}{*}{ Overflow } & Buffer overflow \\
\hline & & Integer overflow \\
\hline & & $\ldots \ldots$ \\
\hline & \multirow{3}{*}{$\begin{array}{l}\text { Manipula- } \\
\text { tion }\end{array}$} & Path manipulation \\
\hline & & $\begin{array}{l}\text { Configuration } \\
\text { manipulation }\end{array}$ \\
\hline & & $\ldots \ldots$ \\
\hline & \multirow{4}{*}{$\begin{array}{l}\text { Access } \\
\text { Control }\end{array}$} & Password crack \\
\hline & & Poor lock \\
\hline & & Race condition \\
\hline & & $\ldots \ldots$ \\
\hline & \multirow{4}{*}{ Leak } & Resource leak \\
\hline & & Memory leak \\
\hline & & Information leak \\
\hline & & $\ldots \ldots$ \\
\hline & \multirow{3}{*}{ File system } & File upload \\
\hline & & File include \\
\hline & & ...... \\
\hline & \multicolumn{2}{|l|}{$\ldots \ldots \ldots \ldots$} \\
\hline
\end{tabular}

Table 3. Representation of Defects

\begin{tabular}{|c|c|c|}
\hline \multirow{22}{*}{ 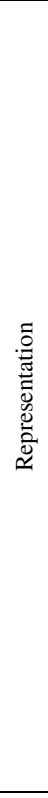 } & \multirow{4}{*}{$\begin{array}{c}\text { String } \\
\text { Manipulation }\end{array}$} & $\begin{array}{l}\text { Misjudgement of length } \\
\text { of string }\end{array}$ \\
\hline & & Format string \\
\hline & & String iteration \\
\hline & & ...... \\
\hline & \multirow{3}{*}{ Semaphore } & Single member field \\
\hline & & Semaphore handle \\
\hline & & $\ldots \ldots$ \\
\hline & \multirow{3}{*}{ Expression } & Expression is always true \\
\hline & & $\begin{array}{l}\text { Expression is always } \\
\text { false }\end{array}$ \\
\hline & & $\ldots \ldots$ \\
\hline & \multirow{4}{*}{$\begin{array}{l}\text { Exception } \\
\text { handle }\end{array}$} & Empty Catch block \\
\hline & & Unhandled exception \\
\hline & & Overly broad throws \\
\hline & & $\ldots \ldots$ \\
\hline & \multirow{4}{*}{ Math } & Confusion of math operators \\
\hline & & Mix of mathematical type \\
\hline & & Divided by 0 \\
\hline & & $\ldots \ldots$ \\
\hline & \multirow{3}{*}{ Constant } & $\begin{array}{l}\text { Unreasonable definition } \\
\text { of constant }\end{array}$ \\
\hline & & Out of bounds \\
\hline & & $\ldots \ldots$ \\
\hline & …...... & \\
\hline
\end{tabular}
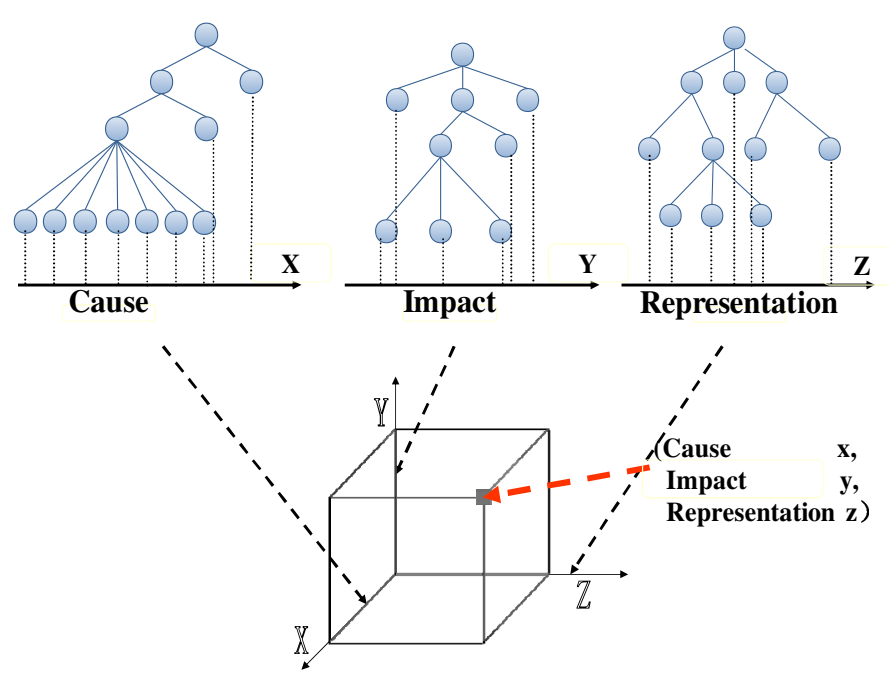

Fig. 2. Three-Dimension-Tree

As we see, this taxonomy could reflect the information and feature of defect in multi-aspects, which is good for the construction of defect detection rules. Besides, it is easy to extend categories in this method. When new defects come out, we should only insert 3 new nodes into 3 trees at suitable positions. 
Nomenclature of Defect Categories. In this taxonomy, we name a kind of defects with a triple that includes the names of its corresponding cause, impact and representation, which is intuitive and applied. Defect's features can be reflected by its name, which is good for defects' modification and statistics. For example, we name the kind of defect that is described in code

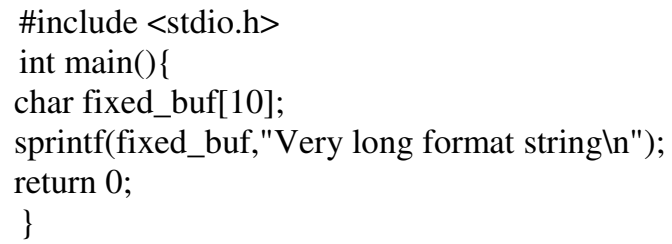

with (Input issues and validation, Buffer overflow, Array out of bounds in Sprintf()).

\section{Case Study}

Classification of CWE Defects. We classify about 110 items of source code defects listed in CWE with new taxonomy, and acquire 146 categories. Tab. 4 illuminates 12 of them. The second column indicates the item of defects that corresponding CWE ID denotes.

Following conclusions can be drawn from Tab. 4:

Table 4. 12 categories of CWE Defects

\begin{tabular}{|c|c|c|c|c|}
\hline \multirow{2}{*}{ id } & \multirow{2}{*}{ CWEID } & \multicolumn{3}{|c|}{ Three-Dimension-Tree Taxonomy } \\
\hline & & Cause & Impact & Representation \\
\hline 1 & 113 & $\begin{array}{l}\text { Input issues and } \\
\text { validation }\end{array}$ & $\begin{array}{l}\text { HTTP response } \\
\text { truncation }\end{array}$ & HTTP Cookie with incredible data \\
\hline 2 & 79 & $\begin{array}{l}\text { Input issues and } \\
\text { validation }\end{array}$ & Cross-site scripting & \\
\hline 3 & 563 & Unsafe code & Dead code & Unused variable \\
\hline 4 & 563 & Unsafe code & Unsafe style & Coverage of independent increment \\
\hline 5 & 242,676 & API errors & Dangerous Function & Mac OS function \\
\hline 6 & 242,676 & API errors & Dangerous Function & Unix function \\
\hline 7 & 404 & Unsafe code & False release & \\
\hline 8 & 404,772 & $\begin{array}{l}\text { Boundary } \\
\text { treatment }\end{array}$ & Unreleased Resource & File unclosed \\
\hline 9 & 103 & $\begin{array}{l}\text { Input issues and } \\
\text { validation }\end{array}$ & Struts errors & validate() error \\
\hline 10 & 104 & $\begin{array}{l}\text { Input issues and } \\
\text { validation }\end{array}$ & Struts errors & No inheritance of Validation \\
\hline 11 & 105 & $\begin{array}{l}\text { Input issues and } \\
\text { validation }\end{array}$ & Struts errors & Missing Validator \\
\hline 12 & 590 & $\begin{array}{l}\text { Input issues and } \\
\text { validation }\end{array}$ & Misuse of memory & $\begin{array}{l}\text { Memory for free() isn't provided by } \\
\text { malloc() }\end{array}$ \\
\hline
\end{tabular}

(1) Defects' detail information can be presented with new taxonomy. For example, the 1 st category of defects is caused by input, and may import the impact of HTTP response truncation. It's representation in code is one HTTP Cookie with incredible data. In addition, the $3 \mathrm{rd}$ and 4 th categories are described as one item of defects in CWE (Code style and quality, ID is 563), but they are greatly different in impacts and representations, and these differences are embodied in new classification; 
(2) The information about platform and language can be presented in new method. For example, the 5th and 6th categories are special for Mac OS and Unix respectively, while the 12 th for $\mathrm{C} / \mathrm{C}++$;

(3) The attribute of representation in new taxonomy increases the intuition of defect. The 9th, 10th and 11th categories are all caused by input issues and validation, and all import Struts errors, but their representations are different, which are validate() error, no inheritance of Validation, and missing Validator. It's more intuitionistic.

Table 5. 10 categories of Fortify Defects

\begin{tabular}{|c|c|c|c|c|}
\hline \multirow{2}{*}{ id } & \multirow{2}{*}{ Fortify Defects } & \multicolumn{3}{|c|}{ Three-Dimension-Tree Taxonomy } \\
\hline & & Cause & Impact & Representation \\
\hline 1 & Cross-site scripting & $\begin{array}{l}\text { Input issues and } \\
\text { validation }\end{array}$ & Cross-site scripting & Poor Validation \\
\hline 2 & Buffer overflow & $\begin{array}{l}\text { Input issues and } \\
\text { validation }\end{array}$ & Buffer overflow & Format String $(\% \mathrm{f} / \% \mathrm{~F})$ \\
\hline 3 & Dead code & Unsafe code & Dead code & Unused variable \\
\hline 4 & SQL injection & $\begin{array}{l}\text { Input issues and } \\
\text { validation }\end{array}$ & SQL injection & Hibernate \\
\hline 5 & Access control & $\begin{array}{l}\text { Access control and password } \\
\text { fail }\end{array}$ & Access control & $\begin{array}{l}\text { Anonymous LDAP } \\
\text { Bind }\end{array}$ \\
\hline 6 & Race condition & Share and race & Race condition & File system access \\
\hline 7 & Memory leak & Unsafe code & Memory leak & $\begin{array}{l}\text { Memory } \\
\text { redistribution }\end{array}$ \\
\hline 8 & $\begin{array}{l}\text { System information } \\
\text { leak }\end{array}$ & Boundary treatment & $\begin{array}{l}\text { System information } \\
\text { leak }\end{array}$ & Missing Catch Block \\
\hline 9 & $\begin{array}{l}\mathrm{J} 2 \mathrm{EE} \\
\text { misconfiguration }\end{array}$ & configuration errors & $\begin{array}{l}\mathrm{J} 2 \mathrm{EE} \\
\text { misconfiguration }\end{array}$ & $\begin{array}{l}\text { Missing Error } \\
\text { Handling }\end{array}$ \\
\hline 10 & $\begin{array}{l}\text { Object model } \\
\text { violation }\end{array}$ & API errors & $\begin{array}{l}\text { Object model } \\
\text { violation }\end{array}$ & $\begin{array}{l}\text { Just one of equals() } \\
\text { and hashCode() Defined }\end{array}$ \\
\hline
\end{tabular}

Classification of Fortify Defects. Fortify is a famous tool for source code security analysis [19]. It could analyze programs in 19 languages. There are more than 300 items of defects in Fortify, and most of them are defined based on the description of defects in CWE and OWASP. We classify 86 items of them with new taxonomy, and get 194 categories. Tab. 5 lists 10 of them, and the set of defects that are described with 3 attributes in each line is only a subclass of Fortify defects in the same line. That is to say, classes derived from three-dimension-tree taxonomy are more refined.

\section{Conclusion}

In this paper, a new taxonomy for source code security defects based on three-dimension-tree is present, which considers the information of defect's cause, impact and representation synthetically. Case studies show that a sound system for classifying source code defects can be established with this taxonomy, and it is also good for the prevention and fixing of software vulnerabilities. 
Upon existing works, we will continue refining classification attributes. The taxonomy in this paper is based on 9 kinds of causes, more than 30 kinds of impacts and more than 150 kinds of representations, but some defects could not be accurately defined, for example, the representations of the 2 nd and 4 th categories in Tab. 4 are blanks. Thus, our next goal is attributes refinement.

\section{References}

[1] Mei, H., Wang, Q.X., Zhang, L., Wang, J.: Software Analysis: A Road Map. Chinese Journal of Computers 32(9), 1697-1710 (2009)

[2] Piessens, F.: A Taxonomy of Causes of Software Vulnerabilities in Internet Software. In: Proceedings of the 13th International Symposium on Software Reliability Engineering, ISSR 2002 (2002)

[3] Aslam, T.: A Taxonomy of Security Faults in the Unix Operating System. Technique report TR-95-09, Department of Computer Science, Purdue University, West Lafayette, USA (1995)

[4] Jiwnani, K., Zelkowitz, M.: Susceptibility Matrix: A New Aid to Software Auditing. IEEE Security and Privacy 2(2), 16-21 (2004)

[5] Landwehr, C.E., Bull, A.R., McDermott, J.P.: A Taxonomy of Computer Program Security Flaws with Examples. ACM Computing Surveys 26(3), 211-254 (1994)

[6] Weber, S., Karger, P.A., Paradkar, A.: A Software Flaw Taxonomy: Aiming Tools at Security. In: Proceedings of the 2005 Software Engineering for Secure Systems, SESS 2005 (2005)

[7] Tsipenyuk, K., Chess, B., McGraw, G.: Seven Pernicious Kingdoms: A Taxonomy of Software Security Errors. IEEE Security \& Privacy 3(6), 81-84 (2005)

[8] Power, R.: Current and Future Danger: A CSI Primer on Computer Crime and Information Warfare. Computer Security Institute, San Francisco (1996)

[9] Krsul, I., Spafford, E., Tripunitara, M.: Computer Vulnerability Analysis. Technique report TR- 47909-1398, Department of Computer Science, Purdue University, West Lafayette, USA (1998)

[10] Wenliang, D., Mathur, A.P.: Categorization of Software Errors that Lead to Security Breaches. In: Proceedings of the 21st National Information Systems Security Conference (1998)

[11] Bishop, M.: A Taxonomy of Unix System and Network Vulnerabilities. Technical Report CSE-95-8, Dept. of Computer Science, University of California at Davis, Davis (1995)

[12] Cohen, F.B.: Information System Attacks: A Preliminary Classification Scheme. Computers and Security 16(1), 26-49 (1997)

[13] Howared, J.D.: An Analysis of Security Incidents on the Internet 1989-1995. Carnegie Mellon University, Pittsburgh (1997)

[14] Killourhy, K.S., Maxion, R.A., Tan, K.M.: A Defense-centric Taxonomy Based on Attack Manifestations. In: Proceedings of the 34th IEEE/IFIP International Conference on Dependable Systems and Networks, DSN 2004 (2004)

[15] Hansman, S., Hunt, R.: A Taxonomy of Network and Computer Attack. Computers and Security 24(1), 31-43 (2005)

[16] DeMillo, R.A., Mathur, A.P.: A Grammar-based Fault Classification Scheme and Its Application to the Classification of the Errors of Tex. Technique report, Department of Computer Science, Purdue University, West Lafayette, USA (1995) 
[17] Bazaz, A., Arthur, A.J.D.: Towards a Taxonomy of Vulnerabilities. In: Proceedings of the 40th Annual Hawaii International Conference on System Science (2007)

[18] Information on, http: / / cwe.mitre.org/

[19] Information on, http: / / www. fortify.com/

[20] Huang, M., Zeng, Q.K.: Research on Classification Attributes of Software Vulnerability. Computer Engineering 36(1), 184-186 (2010) 